A review of a family and the most recent case history in the only male has shown that the cerebellar tumour was verified for sibling I at the age of 29 years by pathological section and an eye tumour was treated by radon seeds. Sibling II - the cerebellar tumour was verified at operation at the age of 23 years and by pathological section at two subsequent operations. The eye tumour presented a similar pathological appearance. Sibling III had a naevus of the limbus of the left eye, which became less apparent after nine years. Sibling IV: The new case was verified by pathological examination at the age of 28 years and after extensive radiation an eye tumour, capillary in type, has slightly progressed in four years.

Acknowledgment: To Dr. K. G. McKenzie my thanks and congratulations for the surgical care and remarkable results that have meant so much to the members of this family. Dr. Eric Linell was responsible for the excellent pathological reports and photographs. Miss Wishart made coloured drawings, which add much to the written description. Also thanks to the Departments of Surgery, Neuro-Pathology, Veterans' Affairs, Ophthalmology, Art and Photography.

\title{
METHODS OF INVESTIGATING EYE MOVEMENTS
}

\author{
BY \\ H. HARTRIDGE and L. C. THOMSON \\ VISION RESEARCH UNIT, MEDICAL RESEARCH COUNCIL, \\ INSTITUTE OF OPHTHALMOLOGY, LONDON
}

OpINIONS differ widely at the present time with regard to the ability of a subject to perform accurate fixation, for whereas some hold that the eye is constantly making rapid oscillations, on the presence of which visual acuity for fine detail actually depends, others hold, on the contrary, that such oscillations are absent in normal subjects and that if these movements were present, far from aiding the perception of detail, they would have the effect of seriously interfering with it. Thus Hering (1899) thought that the irregularities at the contours between the images of objects which are produced by the retinal mosaic are smoothed by chance eye movements. Anderson \& Weymouth (1923), Averill \& Weymouth (1925), and Marshall \& Talbot (1942) have held similar views, and they have extended the consideration to the cases of small differences of size and small defects in alignment. Wright (1942) claims that the eyes are continually performing " twittering " movements, and that in consequence the receptors are able to scan the retinal image in the same way that the bright spot in the cathode ray tube of a television receiver scans the fluorescent screen. He writes, "It is the dimensions of the cones rather than their number 
which is the essential factor in determining the visual acuity." Thus a sparse population will perform as well as or even better than a dense one. Adler \& Fliegelman (1934), as the result of recording the'reflection of a beam of light from a galvanometer mirror which had been attached by surface tension to the sclera of a subject, claimed to have demonstrated the presence of fine oscillatory rotations of the eyeball. Lastly Lord \& Wright (1948) have stated that they find evidence which confirms that of Adler and Fliegelman, because their records show that the subject's eye is oscillating with an amplitude of about thirty minutes of arc.

In contradistinction to these views experiments were performed by Hartridge (1947) which were based on the employment of very precise fixation. Thus the position of the fixation points for rays. of different colour at the fovea appeared to be identifiable within a fraction of a cone unit, and as a rule ophthalmologists do not find that eye movements interfere with observations of the anterior eye structures by means of the slit-lamp, in which magnifications of between fifteen and thirty diameters are normally employed.

\section{Section 2: Problems connected with the Observation of Eye Movements}

A number of different methods have been employed in the past for investigating the movements of the eye. It is not proposed to consider these in detail in this paper, but instead to concentrate attention on optical methods alone, since in the opinion of the authors these are more likely to lead to accurate results than are methods which depend on other principles.

Three main problems have to be faced in designing a satisfactory technique.

(a) Obtaining the necessary accuracy.

(b) The elimination of chance movements of the head.

(c) Eliminating the effects of the heart beat.

With regard to the first problem the precision needed corresponds to a rotation of the eyeball of one minute of arc. This is the minimum precision which should be aimed at. If a method could be devised which provided a precision of one-tenth of this, that is six seconds of arc, it would be advantageous because one cone unit, namely $2 \mathrm{~mm}$. at 10 metres, the standard proposed by one of us elsewhere (1947), corresponds to an angle of 41.236 minutes of arc, and it would be an advantage therefore to be able to express the rotation of the eye in terms of cone units and fractions of a cone unit. If a human eye rotated through an angle of one minute of arc the various parts forming the coats of the 
eye would suffer a rotatory displacement of about $2 \cdot 4 \mu$. The optical method therefore has to satisfy the requirement of being able to provide measurement of such a displacement to at least an accuracy of $2 \cdot 4 \mu$, and if possible to an accuracy of $0 \cdot 24 \mu$.

With regard to chance head movements these are frequently quite large. The precise cause of them is not known. It may be that they are due merely to irregularity in the contraction of the muscles which support the head on the body, and to similar irregularity in the muscles which maintain the position of the body. Another possibility is that the proprioceptive control of the head in relation to the environment lacks high precision. Whatever the cause may be there is little doubt that the head is constantly performing small nodding movements, both lateral and anteroposterial. These are largely counterbalanced by compensatory rotations of the eyeball which, in spite of head rotations, maintain the gaze on external objects. The study of eye rotations during the rotation of a subject, by means of a cinema camera mounted on the same turntable as himself, had already provided us with much information with regard to these compensatory rotations of the eye, but there is little doubt that much remains to be learnt concerning them.

With regard to the effects of the heart beat the eye itself appears to be so arranged in the orbit as to be immune, or almost immune, from the effects produced by variations in blood flow during the various phases of the cardiac cycle. The effects of the heart beat most strongly assert themselves either when apparatus is mounted on the head, attached to a plaster hat, or when attempts are made to immobilise the head by means of pads or clamps externally applied. Because of these circulatory effects in the soft tissues of the head it would appear to be a matter of considerable difficulty to immobilise the head completely. It will be recollected that even the teeth are not in solid contact with the bones of the upper and lower jaws, but that they are inserted into soft tissues which are themselves supplied with blood and therefore pulsating to some extent with the heart beat.

\section{Section 3: The Choice of Suitable Eye Structure}

It is obvious that theoretically any structure accessible to light may be utilised for recording optically the rotations of the eyeball. It is therefore largely the practical considerations which decide what structure provides advantages over others. It is not possible to contrast all the possible structures which are available. A few of the more important only will be considered in this paper.

The cornea.-This structure has two advantages. It is readily 
accessible and its anterior surface reflects light in the same way that a convex mirror of short radius of curvature reflects light. It was for these reasons that at the suggestion of one of us Vernon made use of the reflection from the cornea, when studying the eye movements which occur during reading.

The corneal reflection has one disadvantage, namely that the movement of the reflected image of the light source is not as great as the actual movement of the anterior surface of the cornea itself, but is about half the movement. In order therefore to measure the rotations of the eye with a precision of one minute of arc it would be necessary to be able to measure the movement of the corneal reflection with a precision of about $1 \cdot 8 \mu$. There is another disadvantage in using the corneal reflection, namely that deflections may take place owing to alterations in the local thickness of the lachrymal fluid. This is very likely to occur if the reflected image is taken off the eyeball near the margin of either the upper or the lower lid, or if any factor connected with the observations is causing lacrymation.

The iris.-This structure in subjects whose irises are not deeply pigmented provides an admirable object for photography, the obvious disadvantage attending its use being the constant alterations in the diameter of the pupil which are all the time taking place in normal subjects. These difficulties may in large measure be avoided by paralysing the iris, either in its dilated condition by means of atropine or in its contracted condition by means of eserine. 'The line of demarcation between the edge of the iris and the pupillary aperture under either of these treatments provides a sharp edge by means of which the necessary precision should be obtainable. There is one disadvantage however, that it is not easy to illuminate the substance of the iris brightly without at the same time flooding the eye with light. To some extent this difficulty can be offset by directing the illuminating beam towards the subject's blind spot.

The sclera.-The sclera may be utilised under three conditions.

(a) Where the scleral vessels are partially engorged with blood, so that they provide the necessary landmarks for photography.

(b) Where a suitable part of the sclera has been tattooed.

(c) Where a suitable foreign body has been placed in contact 'with the sclera.

Adler and Fliegelman applied a plane mirror to the sclera allowing it to adhere by surface tension. The disadvantage of this technique was that rocking of the mirror could take place owing to the fact that the sclera was curved but the mirror flat. One method of overcoming this difficulty would be to employ a concave lens, the concavity having the same radius of curvature as that 
of the sclera, the other side of the lens being polished flat or to a suitable curvature and made reflecting by means of aluminium, silver or rhodium. Such a lens-mirror should not be found to rock during the rotations of the eye. Another method has recently been used by Barlow, namely to apply small drops of mercury to the sclera, and to use these as landmarks. The only difficulty which is likely to be met with here is that the mercury droplets may be slowly swept along in the lacrymal fluid which is emerging under the border of the upper lid and is flowing towards the inner canthus of the eye.

The retina.-The retina would appear to have obvious advantages over all the structures which have been previously mentioned, owing to two factors; $(a)$ it is the structure which is vitally concerned with movement because it is the movement of the image of the lens system relative to the retina with which we are concerned when studying eye movements, and $(b)$ due to the fact that the retina rotates in one direction, when the lens system is rotated in the opposite direction, the apparent movement is double that obtained when making observations on the superficial eye structures which have been mentioned above. The difficulties likely to be met with in practice are those connected with the illumination of the retina, and in the provision of a sufficiently fast film for adequately recording the somewhat feeble light which re-emerges through the pupillary aperture. By choosing a subject with slight retinal pigmentation and by directing the illuminating beam at the blind spot, which is as a rule paler than the rest of the retina, it should be possible to obtain sufficient illumination without at all seriously disturbing the retina and preventing adequate fixation.

The contact glass. - It is obvious that if a contact glass could be fitted to the subject's eye, so well-fitting that it moved precisely with even the smallest rotations of the eye, and not at the same time causing painful sensations, it would go a long way to providing the solution of many of the problems indicated in earlier parts of this section, thus the artificial cornea could be given a curve of a much smaller radius than the real cornea, the consequence of which would be that the ray reflected from its surface would undergo a bigger movement for a bigger rotation of the eye than the subject's real cornea. A long-sighted subject is preferable for such a method, because in order to correct his long sight a contact lens with a more highly curved cornea would be indicated. Secondly it would be possible to mount a galvanometer mirror on such a contact lens just to one side of the cornea. There would be no possibility of such a mirror rocking during eye rotations, as might be the case if the mirror were attached directly to the sclera. Thirdly it would be possible to engrave suitable 
marks either on the centre of the artificial cornea or to one side of it, or on the artificial sclera, at will, but as indicated above the method would only succeed if the contact lens precisely fitted the curvatures of the subject's eyebahl, so that there would be complete absence of "backlash" between the eyeball and the contact lens.

Reference has already been made to the fact that whereas the corneal reflection moves less than the cornea itself, for a given rotation of the eye, the apparent rotation of the retina is double that of the cornea, because it is being viewed via the lens system of the eye. It may be useful to summarise the efficiencies of the other structures mentioned above.

TABLE

\begin{tabular}{|c|c|c|c|c|c|c|c|}
\hline \multirow{2}{*}{\multicolumn{3}{|c|}{$\begin{array}{l}\text { Structure } \\
\text { Corneal reflection }\end{array}$}} & \multirow[b]{2}{*}{.. } & \multirow[b]{2}{*}{$\ldots$} & \multirow[b]{2}{*}{$\ldots$} & \multicolumn{2}{|r|}{ Efficiency } \\
\hline & & & & & & $\ldots$ & $0.5-0.4$ \\
\hline Iris & $\ldots$ & $\ldots$ & $\ldots$ & $\ldots$ & ... & $\ldots$ & $1 \cdot 0$ \\
\hline Sclera & $\ldots$ & $\ldots$ & $\ldots$ & $\ldots$ & $\ldots$ & $\ldots$ & $1 \cdot 0$ \\
\hline Retina & $\ldots$ & $\ldots$ & ... & $\cdots$ & ... & $\cdots$ & $1 \cdot 9-2$ \\
\hline Scleral & airro & .. & $\ldots$ & $\ldots$ & $\ldots$ & $\ldots$ & $3-4$ \\
\hline
\end{tabular}

It will be seen that according to the above table the scleral mirror provides the most efficient optical arrangement.

\section{Section 4: Some Suitable Optical Arrangements}

There are broadly two optical methods which may be adopted for determining the eye movements. One consists of photographing the movements after suitable magnification, by means of a cinema or other camera. The other consists in utilising two or more photoelectric cells and amplifying and recording the currents which are generated in these. The relevant pros and cons of these methods are difficult to assess but both should give satisfactory results. The first method has been used recently by Hartridge and Thomson, the latter method is in use by Lord and Wright. When their method is used the subject lies on his back, his head resting in a ring; suitable supports are placed on either side of the head; a mouth plate is also employed. In these various ways head movements are restricted. Actual head movements are recorded by means of an artificial cornea which is attached to a suitable fitting which clamps on one of the front teeth. Both head and eye movements together are recorded by causing rays of light from an ultra-violet lamp after reflection in the corneal surface to fall on two photo cells, in front of which are placed knife edges in order to cut off part of the reflected beam. When 
the cornea moves the beams of light are deflected. In consequence more or less light falls on the photo cells. The voltages on the cells after suitable amplification cause excursions of the fluorescent point of light in cathode ray tubes, and these are recorded photographically in the ordinary way. Continuous records are obtainable. So far they have not devised apparatus which will record both head and head and eye movements at the same time, so that they have not obtained eye movements independently of head ones.

The present method used by Hartridge and Thomson consists of a low-powered microscope, which is attached to a plaster of Paris hat which fits on the subject's head. This hat also carries

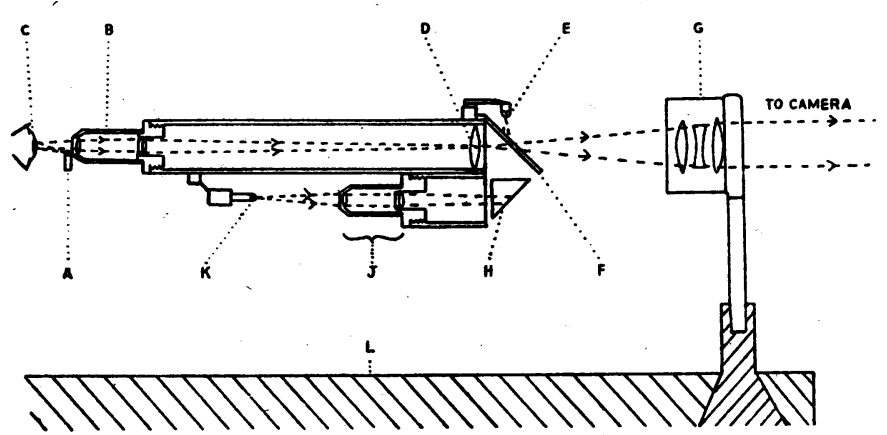

FIG. 1.

A plan of the original apparatus used by Hartridge and Thomson to measure movements of the eye.

A, light source to provide the corneal reflection; $B$, microscope objective; $C$, cornea; $D$, field lens; $E$, reference source; $F$, sloping glass plate to introduce reference image ; $G$, collimator; $H$, right-angled prism ; $J$, objective for the introduction of the fixation spots; $K$, source of light for fixation; $\mathrm{L}$, optical bench.

the light source for illuminating the cornea, a reference source which is photographed alongside the magnifying reflection of the corneal image and a fixation light source, which by suitable optical means provides the subject with two bright points of light, on either of which at will he may preserve fixation. The low-powered microscope has such a focal length that the source of light, after reflection in the cornea and re-magnification, has approximately the same dimensions as the reference source. The latter is introduced into the light beam by two reflections at an unsilvered glass plate. In consequence two images of the reference source are seen by the camera. This duplication is found to assist the subsequent alignment and measurement of the photographic film. The cine camera which takes up to sixty frames per second is focused at 
infinity, and the rays from the low-powered microscope attached to the subject's head, after passing through a field lens, enter a collimator lens which is attached to an optical bench. The optical bench to which the camera is attached is fixed to an independent

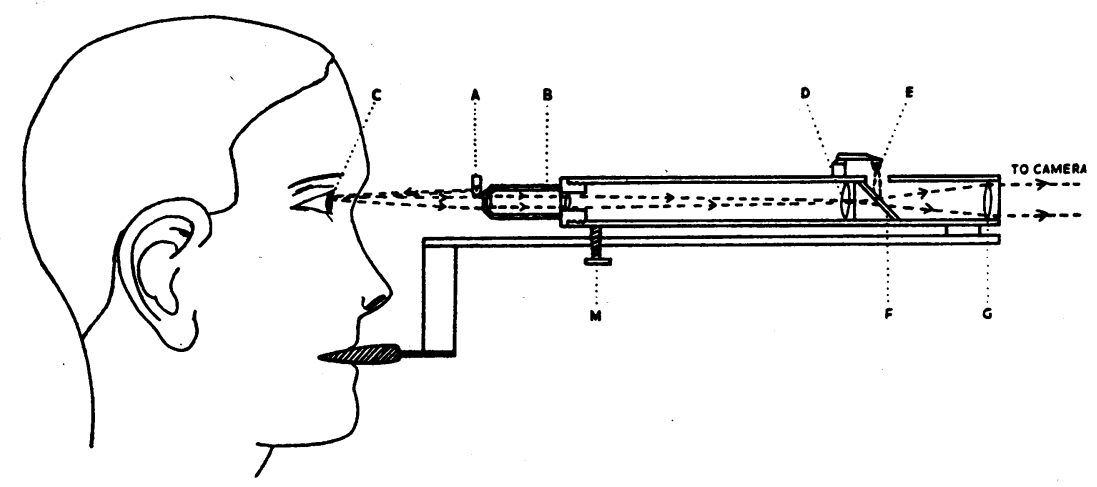

FIG. 2.

A plan of the new apparatus for measuring eye movements. Lettering as for Fig. 1, except that $M$ indicates a screw for vertical height adjustment.

table, so that vibrations set up by the motor of the camera are not conveyed to the apparatus attached to the hat of the observer.

A new type of apparatus has recently been devised by Hartridge and Thomson, namely to hang from a suitable counterpoise a frame which carries the microscope, the reference lights and the

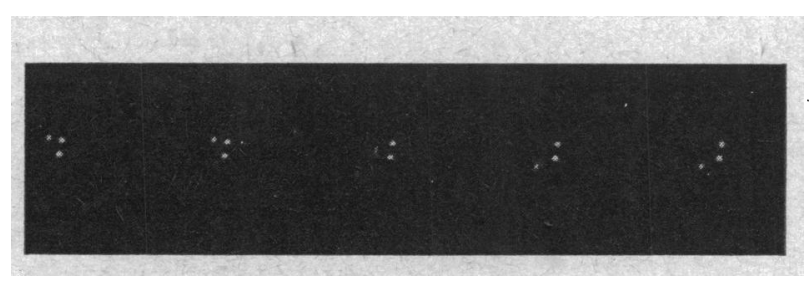

FIG. 3.

Five frames from a cine film taken at a speed of 64 frames a second, showing an intentional eye movement. The central frame shows the two reference images lying beside the moving corneal reflection.

corneal light source. It also carries a mouth plate which fits on to the teeth of the subject. The counterpoise is so arranged that the apparatus tends neither to rise nor to fall, but can be freely rotated in all directions. The optical apparatus is so disposed that the subject can observe uninterruptedly suitable fixation points of light which are attached to a wall beyond the apparatus. This 
apparatus retains the advantage of the previous one, of enabling measurements of eye movements to be obtained which are quite independent of head movements. It has three other advantages. In the first place no constraint need be placed on head movements

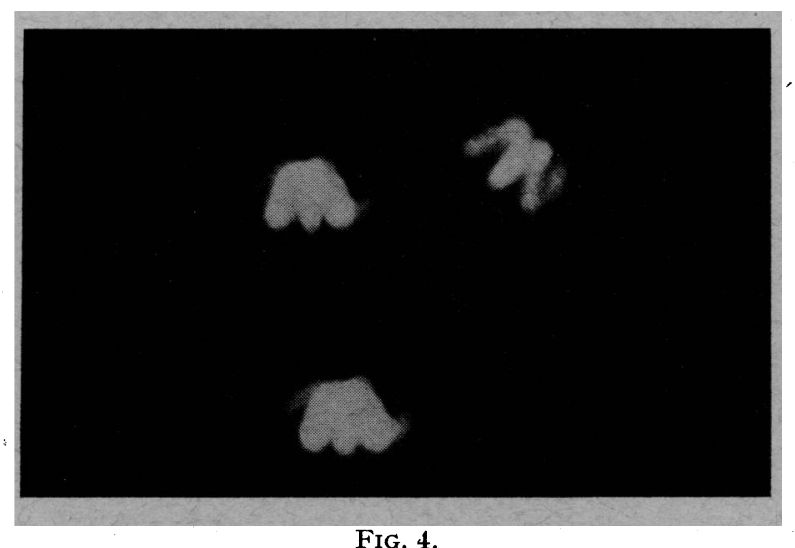

FIG. 4.

An enlarged image of a single cine frame from a strip of film taken during fixation of the eye. The corneal is the unpaired image on the right.

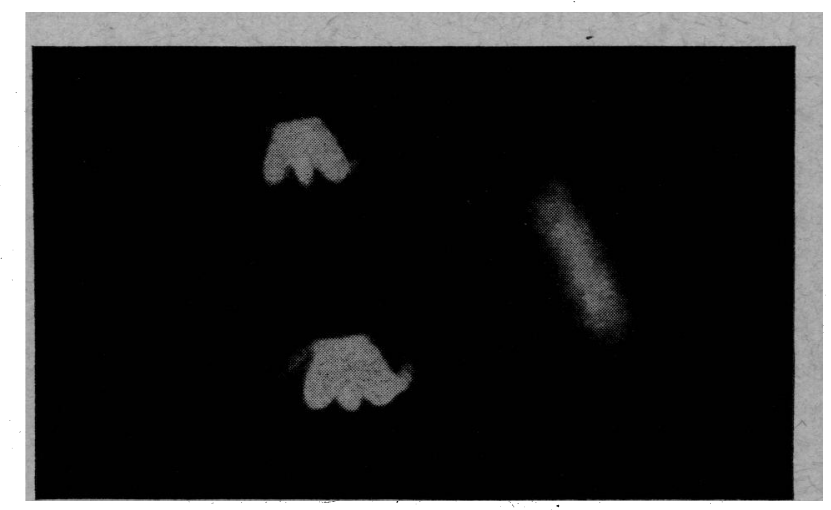

Fig. 5.

An enlarged image of a single cine frame from a strip of film taken during an intentional movement of the eye.

and no difficulties arise through attempting to immobilise the subject's head. In the second place the head movements relative to the camera may be determined by the movements performed by the images of the reference lights. Lastly since the optical equipment is not fastened to a plaster hat which rests on the subject's 
head, fluctuations in vascularity do not affect the position of the apparatus in relationship to the eye.

A diagram of the old apparatus employed by Hartridge and Thomson and a diagram of the new apparatus now under construction are shown in Figs. 1 and 2. In Fig. 3 is shown an enlargement of a short length of cine film showing an intentional vertical eye movement. In Fig. 4 is shown an enlarged image of a single corneal reflection. Such fine definition would be impossible if the eye were performing very rapid vibratory rotations. Fig. 5 shows an enlarged image of a corneal reflection photographed whilst the eye was performing an intentional eye movement.

One millimetre on Figs. 4 and 5 corresponds to a displacement of the image on the retina equal to $28 \mu$, that is, to 8.3 cone units.

\section{Section 5: The Examination of the Photographs}

Three methods have so far been used for examining the cine film : (a) The detailed examination of single frames with a lowpower microscope; $(b)$ the projection of lengths of film on to a screen at one-quarter the speed at which they were taken; $(c)$ the measurement of selected frames, using a microscope which has been fitted with a Leitz mechanical eyepiece micrometer. The latter method was found to be very laborious.

A fourth method, which is to be tested as soon as the necessary apparatus is available, consists in the preparation of an enlarged negative of one frame of the film and then the projection of the magnified images of other frames on to this enlargement, the magnification used being the same as that employed during the making of the enlargement. This method requires a special microscope having two mechanical stages, one about $200-250 \mathrm{~cm}$. above the other, between the two being mounted a projection lens of short focal length. On the lower stage is placed the film strip and on the upper one the enlarged negative. The projection lens, which magnifies about 8 times, now produces an image of the film in the plane of the enlarged negative.

Using the horizontal and vertical adjustments the corresponding parts of the images of the filaments of the reference lights are now superposed, and adjustments are made until an exact fit is obtained. Now if the frame and the enlargement are alike, the consequence will be that when the images of the reference light are in exact superposition the images of the corneal reflection will be in exact superposition also. If on the contrary they are unlike, owing to a rotation of the eye, then a movement either of the film strip or of the enlargement will be required in order to superpose first the images of the reference light and then that of the corneal light, and this movement will be a measure of the eye movement which 
has taken place between the exposure of the frame from which the enlargement has been made and that now being examined. It is hoped that this method will enable the films to be examined with the required accuracy at a rate of about one frame every two or three minutes.

\section{REFERENCES}

Adler, F. H. and Fliegelman, M. (1931).-Arch. Ophthal., N.Y., N.S., Vol. XII, p. 475.

Anderson, E. E. and Weymouth, F. W. (1923).-Amer. Jl. Physiol., Vol. LXIV, p. $56 \mathrm{I}$.

Averill, H. L. and Weymouth, F. W. (1925).-Jl. Comp. Psychol., Vol. V, p. 147.

HARTRIDGe, H, (1947).-Phil. Trans. B., 232, 592, p. 537.

Hering, E. (1899).-Ber. d, math.-phys. Kl. d. K. Sachs. Gesellsch. d. Wissensch. zu Leipzig, p. 18.

LORD, M. and WRIGHT, W. D. (1948).-Personal communication.

Marshall, W. M. and Talbot, S. A. (1942).-Biol, Symp., Vol. VII, p. 117,

WRIGHT, W. D. (1942).- TheRefractionist, Vol. XXXI, p. 53.

TISSUE CULTURES OF MOUSE LENS EPITHELIUM BY

IDA MANN

From the Laboratories of the Imperial

Cancer Research Fund, London

IT has been pointed out by $\mathrm{Kirby}^{1}$ that if an attempt is made to grow the lens in tissue culture the only cells which survive and multiply are those of the subcapsular epithelium. This is to be expected since the lens fibres are fully differentiated and cannot divide. Kirby's preparations were made with the lenses of chicks. In the present experiments an attempt was made to grow mammalian lens epithelium in tissue culture.

\section{EXPERIMENTS}

Mice were used, mostly of the inbred strain known as $\mathrm{C} 3 \mathrm{H}$. Lenses of young mice up to 10 days old were used first but proved difficult to plant out without infection. Lenses from embryo mice of the same strain were then tried,with greater success. The embryos used were in the later stages of the pregnancy and good results were obtained with full term embryos taken just before birth. With care uninfected cultures could be obtained in about 60 per cent. of the trials. About 70 cultures were made in all.

The culture medium which gave the best results had the following formula:- 